\title{
Experimental and theoretical studies of agglomeration effects in multi-walled carbon nanotube-polycarbonate melts
}

\author{
S. Richter ${ }^{1}$, M. Saphiannikova*1, D. Jehnichen ${ }^{1},{\text { M. } \text { Bierdel }^{2} \text {, G. Heinrich }}^{1}$ \\ ${ }^{1}$ Leibniz Institute of Polymer Research, Hohe Str. 6, 01069 Dresden, Germany \\ ${ }^{2}$ Bayer Technology Services GmbH, 51368 Leverkusen, Germany
}

Received 7 July 2009; accepted in revised form 2 September 2009

\begin{abstract}
In this study we report on morphological and rheological characterization of multi-walled carbon nanotube (MWNT)-polycarbonate composites produced by injection molding. The main focus is to carry out nonlinear viscoelastic experiments that allow following the structural rearrangements of carbon nanotubes in the polycarbonate melt. Small angle X-ray scattering reveals only a slight orientation of MWNTs in the as-received samples, i.e. after application of extremely high shear rates. Thus, the main structural effect observed during the stress growth experiment is the breakage of MWNT agglomerates. To study this effect in detail a flocculation experiment, in which the sample undergoes oscillatory deformation first at a small strain amplitude in the linear regime succeeded by higher amplitudes in the nonlinear regime, has been carried out. The agglomeration process manifests itself in an increase of the storage and loss moduli in the linear regime, whereas the deagglomeration process does vice versa. The corresponding effects can be described in the frame of a superposition approach that takes into account the stress contribution of the polycarbonate matrix, the hydrodynamic reinforcement due to embedded nanotubes and the viscoelastic stress due to the presence of a MWNT-network.
\end{abstract}

Keywords: nanocomposites, rheology, modeling

\section{Introduction}

In the last two decades, polymer nanocomposites have drawn serious attention both from industry and academic research. On the one hand, significant improvements in various properties at low filler concentration are very attractive to industry. On the other hand, researchers show growing interest in studying the influence of nanoscopic filler particles on thermal, electrical and mechanical properties of the composites [1].

A theoretical description of the viscoelastic properties of filled polymer melts represents a great challenge. Here, one must take into account that most filler particles possess an active surface, tend to build agglomerates and at high loadings even a net- work-like superstructure [2-4]. This network is very fragile and can be easily broken in external flow fields. However, in the quiescent state the filler particles agglomerate again and restore slowly a network superstructure. Additionally to the agglomeration process, one observes noticeable orientation effects, as the particles of interest are highly anisometric and thus tend to orient along the flow direction $[5,6]$. The process of orientation is partly reversible because in the quiescent state the filler particles slowly return towards an isotropic orientation state. It is important to note that both agglomeration and orientation processes have much shorter relaxation times in the presence of flow than in the quiescent state [7]. 
Recently, we proposed a superposition approach for description of the viscoelastic properties of low density polyethylene nanocomposites filled with layered double hydroxide particles [8]. In that study the total stress was represented by a superposition of the viscoelastic stress arising in the hydrodynamically reinforced polymer matrix and a stress contribution due to the presence of filler network structure. In the present paper we report on the first attempts to apply the superposition approach to polycarbonate (PC) filled with multi-walled carbon nanotubes (MWNTs). All rheological measurements were carried out with samples produced by injection molding technique at Bayer AG (Germany).

Taking into account excluded volume interactions, the overlap concentration for nanotubes with the persistence length of about $1 \mu \mathrm{m}$ is estimated to be 0.005 volume fraction ( $1 \mathrm{wt} \%$ ) which is in agreement with the experiment [9]. Above the overlap concentration, one obtains a semidilute solution of increasingly entangled nanotubes, i.e. a highly elastic network structure. The boundary between dilute and semidilute regimes is often referred in the literature as the rheological percolation threshold [10]. It is well-known that low values of percolation threshold can be found only in the case of a good dispersion of carbon nanotubes (CNTs) in a polymer matrix. One simple way to control the quality of dispersion is to measure the frequency dependence of storage modulus [3]. Already nanocomposites with $2 \mathrm{wt} \%$ of well-dispersed CNTs exhibit a low-frequency plateau which extends to higher frequencies with further increase of CNT concentration. This plateau was observed in other, at first sight, quite different systems including filled polymer melts [11, 12], molecular glasses [13] and colloidal suspensions [14]. In all these systems, the particles interact with each other attractively, which causes a non-equilibrium transition from a liquidlike to a solid-like state at high particles densities.

To gain insight into the physics of this process, we have undertaken a relatively simple nonlinear viscoelastic investigation, herein after referred to as the flocculation experiment. In this experiment the structural changes in the filled polymer melt are followed by measuring the time evolution of storage and loss moduli after application different strain amplitudes to the sample, below and above the critical strain. We already reported on the flocculation experiment in our previous paper [8] about low density polyethylene nanocomposites filled with layered double hydroxide particles. Here, we make a step further and try to reproduce the measured data theoretically, with the help of a superposition approach in which the contributions from the polymer matrix and the CNT phase are accounted for separately.

\section{Materials and sample preparation}

Polycarbonate, Macrolon ${ }^{\circledR}$ type $2805,\left(T_{G}=145^{\circ} \mathrm{C}\right.$, melt flow index $(\mathrm{MFI})=10 \mathrm{~g} / 10 \mathrm{~min}$ at $300^{\circ} \mathrm{C}$, $\rho=1.2 \mathrm{~g} / \mathrm{cm}^{3}$ ) was produced by Bayer Material Science AG, Leverkusen, Germany. Values for the molecular weights and the polydispersity index of this polymer measured by size exclusion chromatography (polystyrene calibration, chloroform solvent) are as follows: $M_{w}=4.40 \cdot 10^{4} \mathrm{~g} / \mathrm{mol}, M_{n}=$ $1.19 \cdot 10^{4} \mathrm{~g} / \mathrm{mol}$ and polydispersity index $(\mathrm{PDI})=$ $\left(M_{w} / M_{n}\right)=3.7$. The polymer is of linear structure and amorphous according to the manufacturer specification.

The multi-walled carbon nanotubes used in this study were Baytubes ${ }^{\circledR}$ from Bayer Material Science AG with max. 5\% inorganic impurities. The mean diameter is of about $13-16 \mathrm{~nm}$ and the tube length varies between 1 and $10 \mu \mathrm{m}$. Thus, the aspect ratio of about 50 can be expected for this polydisperse product.

The MWNT-polycarbonate composites were produced by the melt-mixing process in a twin screw extruder (ZSK 26 MC from Coperion Werner \& Pfleiderer, Stuttgart, Germany) in one processing step. Both, the polycarbonate and the MWNTs were fed into the main feeder of the extruder. The total mass flow was in the range of 24 to $27 \mathrm{~kg} / \mathrm{h}$ depending on the MWNT content which was varied in the range between 3 and $5 \mathrm{wt} \%$. The screw speed was set to $400 \mathrm{rpm}$. All barrels of the twin screw extruder were set to a heating temperature of $280^{\circ} \mathrm{C}$ except the barrels of the melting zone which were set to 160 and $205^{\circ} \mathrm{C}$. After compounding, the MWNT-polycarbonate composites were cooled down in a water bath and were finally granulated with a pelletizer. The granulates were used to prepare disk-shaped CNT-polycarbonate plates with a diameter of $80 \mathrm{~mm}$ and a thickness of $2 \mathrm{~mm}$ by injection molding technique at the Thermoplastic Testing Center of Bayer Material Science AG, Leverkusen, Germany. During the injection molding the 
melt temperature was held at $340^{\circ} \mathrm{C}$, the mold temperature was held at $120^{\circ} \mathrm{C}$. The flow rate during the filling process was set to approximately $5 \mathrm{~cm}^{3} / \mathrm{s}$.

To check the long-term stability of samples at elevated temperatures, thermogravimetric analysis has been carried out. The pure PC samples as well as those filled with $5 \mathrm{wt} \%$ CNTs were found to be very stable, exhibiting the weight loss less than $0.5 \%$ after 4 hours annealing at $230^{\circ} \mathrm{C}$.

\section{Sample characterization}

\section{1. TEM analysis}

The ultra-thin sections for transmission electron microscopy (TEM) were cut both near the surface of the injection-molded plate and in its interior by microtome at room temperature. The images were taken by Tecnai 20 (FEI Company, Hillsboro, Oregon, USA). The TEM image taken from the interior of the injection-molded plate shows a fine disper-

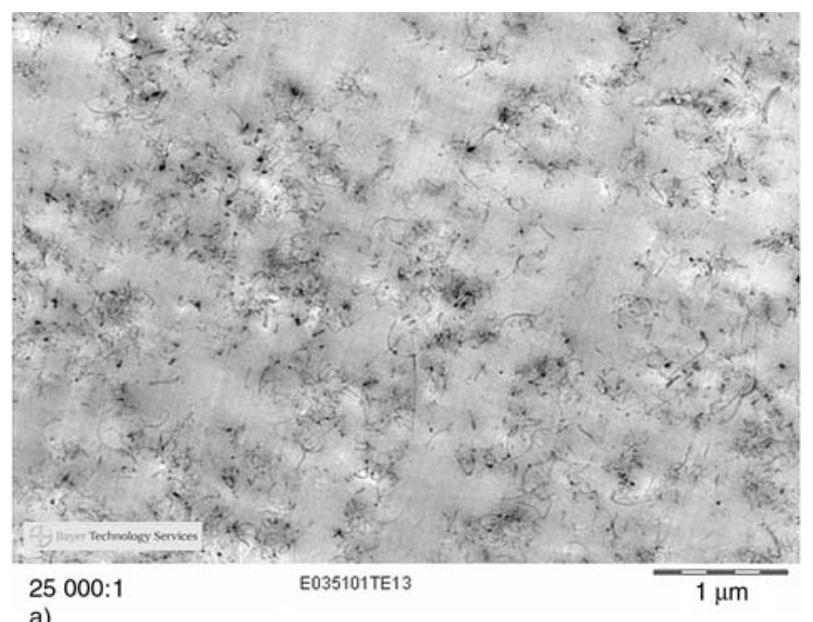

a)

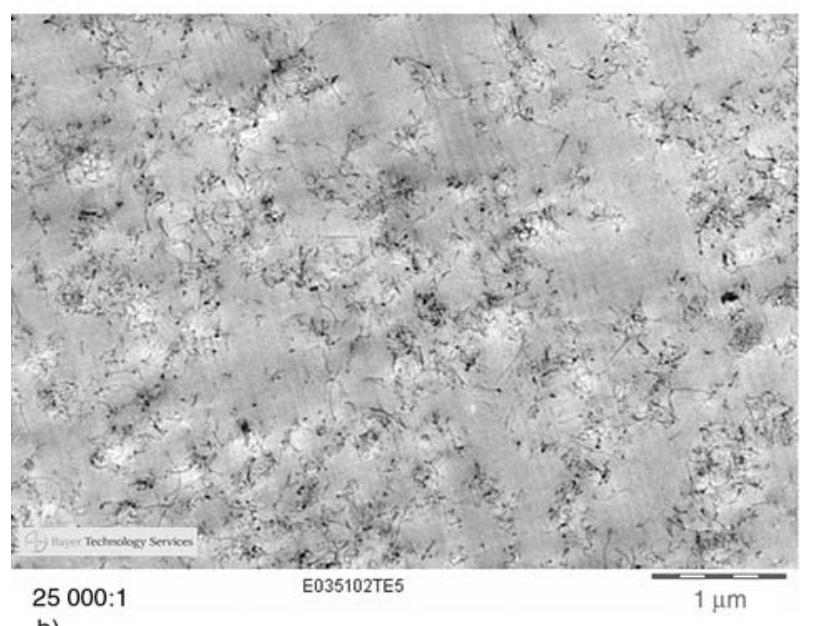

b)

Figure 1. a) TEM picture of the sample as-received,

b) TEM picture of the sample annealed one hour at $300^{\circ} \mathrm{C}$ sion of MWNTs with the presence of small clusters (Figure 1a). The size of the MWNT clusters is estimated to be less than $200 \mathrm{~nm}$. A similar TEM image has been obtained near the sample surface (not shown here). With the naked eye, one sees no orientation of MWNTs in the injection-molded plates obtained at process conditions mentioned above. Also, it is difficult to find noticeable changes in the size of clusters after one hour annealing of the sample at the elevated temperature of $300^{\circ} \mathrm{C}$ (Figure $1 \mathrm{~b}$ ), although the long-term annealing leads to considerably higher storage and loss moduli as will be shown further (see Flocculation experiment).

\section{2. $X$-ray scattering analysis}

The wide angle X-ray scattering (WAXS) pattern for the pure MWNT powder has been measured over $2 \theta=2 \ldots 60^{\circ}$ using XRD $3003 \Theta / \Theta$ (SeifertFPM Freiberg/Sa.) with $\mathrm{Cu}-\mathrm{K}_{\alpha}$ radiation $(\lambda=$ $0.154 \mathrm{~nm}$, monochromatization by primary multilayer system). The WAXS patterns for the pure and filled with $5 \mathrm{wt} \%$ MWNT polycarbonate have been measured over $2 \theta=0 \ldots 40.5^{\circ}$ using X-ray diffractometer P4 (BRUKER axs Karlsruhe, Germany) with $\mathrm{Cu}-\mathrm{K}_{\alpha}$ radiation $(\lambda=0.154 \mathrm{~nm}$, monochromatization by primary graphite crystal). The small angle X-ray scattering (SAXS) patterns for the filled polycarbonate have been measured using a self-constructed 3 fold pin-hole system with a RIGAKU rotating anode generating $\mathrm{Cu}-\mathrm{K}_{\alpha}$ radiation (monochromatization by primary OSMIC confocal optic). The scattering range over $2 \theta=0.065$ $\ldots 2.5^{\circ}(d \approx 135 \ldots 3.5 \mathrm{~nm})$ has been explored by a marCCD X-ray detector system (Mar USA, Inc.). The primary beam stop of $2 \mathrm{~mm}$ diameter has been positioned $1650 \mathrm{~mm}$ away from the sample.

The WAXS patterns of pure polycarbonate do not contain any crystalline reflections (Figure 2a, unfilled symbols). Also, no particle scattering, typical for polycrystalline samples, has been found in the SAXS measurements of pure polycarbonate (Figure 2b, unfilled symbols). Thus, both scattering methods confirm that polycarbonate is amorphous. To check the state of PC orientation in the injection-molded plates, the WAXS patterns were measured at three different positions on the same plate at the direction perpendicular to the plate plane and along the injection molding direction (see Fig- 

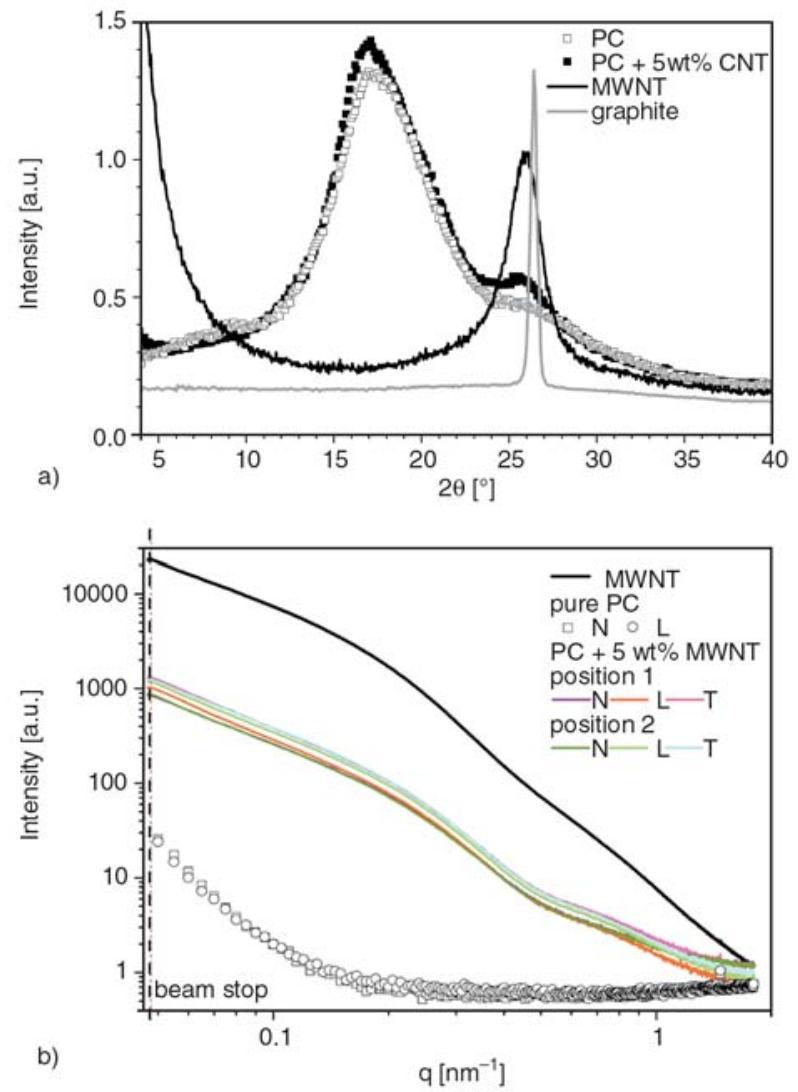

Figure 2. a) Typical WAXS curves for pure polycarbonate and polycarbonate filled with $5 \mathrm{wt} \%$ MWNTs. For comparison, the WAXS curves for pure MWNT powder and pure graphite are shown. b) SAXS curves for pure polycarbonate, measured at two transmission directions, and polycarbonate filled with $5 \mathrm{wt} \%$ MWNTs, measured at the positions 1 and 2 at three transmission directions. For comparison, the SAXS curve for pure MWNT powder is shown.

ure 3). At all positions and directions a wide halo was observed (not shown here). On the WAXS curves (Figure 2a) this halo manifests itself as a wide peak at about $2 \theta=17-18^{\circ}$, corresponding to a length of about $0.51 \mathrm{~nm}$ in the real space. Such a halo is typical for amorphous scattering from organic polymers. Its form is nearly spherical and hence only negligible orientation of the PC has been detected in the injection-molded samples.

In the WAXS patterns of filled systems one observes an additional peak at about $2 \theta=26^{\circ}$. To find a nature of this peak, we performed the WAXS measurement with a pure MWNT powder (Figure $2 \mathrm{a}$, black curve) and compared it with the measurement on pure graphite (Figure 2a, gray curve). The sample of graphite exhibits a sharp peak at $26.6^{\circ}$ originating from the scattering on the stacked graphite layers that have a $0.335 \mathrm{~nm}$ distance. The

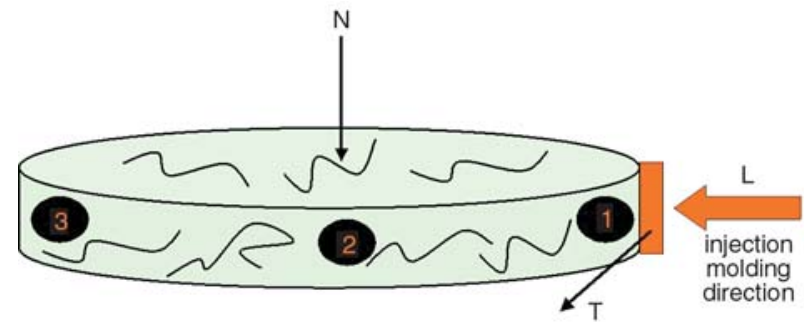

Figure 3. Disk-shaped plate, injection-molded from a side. Here $(\mathbf{N})$ is the direction normal to the plate surface, $(\mathbf{L})$ is the longitudinal direction parallel to that of injection molding, $(\mathbf{T})$ is the transverse direction which is perpendicular to the directions $(\mathbf{N})$ and $(\mathbf{L})$. The samples for the X-ray studies were cut from the plate near its edges at the positions 1, 2 and 3.

MWNT peak is shifted to a slightly smaller angle of $2 \theta=26.0^{\circ}$ compared to the graphite peak and is noticeably wider than the latter. This means that the peak in MWNT powder originates from the scattering between the MWNT walls that are about $0.342 \pm 0.15 \mathrm{~nm}$ from one another.

To check the state of MWNT orientation in the injection-molded plates, the SAXS patterns were measured at two different positions on the same plate (near the injection point and $90^{\circ}$ degrees from it) at three different directions as shown in Figure 3. When the transmission direction is chosen to be perpendicular to the plate surface ( $\mathbf{N}$ direction in Figure 2), one observes a spherical halo in the SAXS patterns for the filled system (Figure 4a). However, in the two other directions, the halo appears to be slightly elliptical that together indicates on slight orientation of MWNTs in the plane of the injection-molded plate. The curves accumulated at two different positions are found to be very similar (Figure 2b) and thus we expect the same degree of MWNT orientation at both positions.

\subsection{Linear viscoelastic analysis}

All oscillatory shear experiments described in this paper were conducted on a controlled strain rate rheometer (ARES rheometer, Rheometrics Scientific) using the plate-plate geometry (diameter of $25 \mathrm{~mm}$, gap between the plates 1-2 mm) in a nitrogen environment. Most studies were performed at $230^{\circ} \mathrm{C}$, although some of linear viscoelastic measurements were conducted in the range $200-300^{\circ} \mathrm{C}$ to allow the time-temperature superposition. All test specimens were cut from the injection-molded 
0

a)
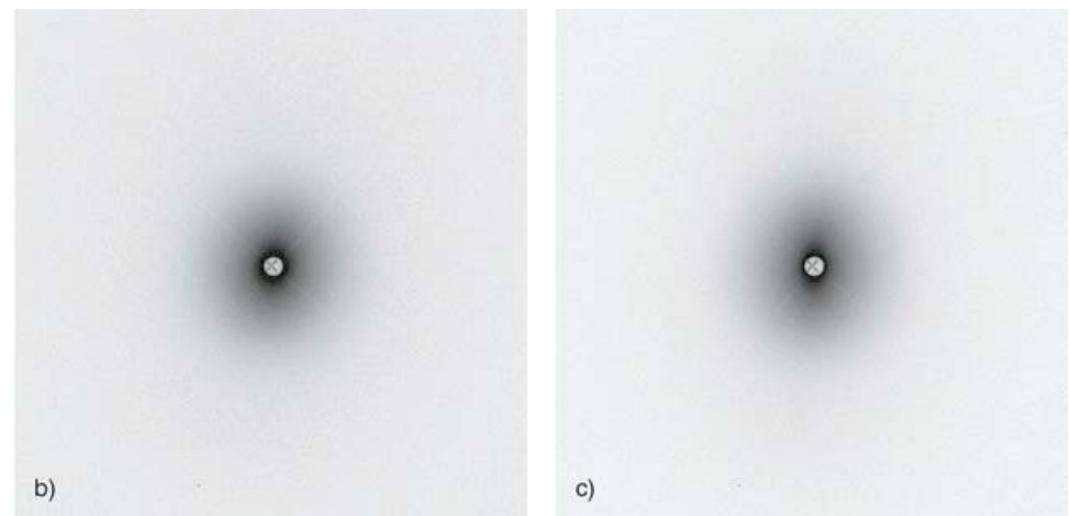

Figure 4. SAXS 2D-pattern for the nanocomposite in different transmission directions: normal (N) to the surface of the plate (a), longitudinal $(\mathbf{L})=$ parallel to the injection molding direction (b), transversal $(\mathbf{T})=$ perpendicular to the injection molding direction (c). The sample was cut at position 1 near the injection molding point. All three directions are depicted in Figure 3.

plates. For the linear and nonlinear viscoelastic measurements, except the 'flocculation' experiment, all the samples were equilibrated in the rheometer after loading at the desired temperature for 15 min prior to testing.

To determine the boundary of linear regime, the strain amplitude sweep tests have been carried out at a constant frequency of $10 \mathrm{rad} / \mathrm{s}(\approx 1.6 \mathrm{~Hz})$ and temperature $230^{\circ} \mathrm{C}$. Figure 5 a presents the strain dependencies of the storage modulus, $G^{\prime}$, for the pure polycarbonate and two composite systems filled with 3 and $5 \mathrm{wt} \%$ CNTs. It is apparent that the unfilled polycarbonate melt exhibits linear behavior up to much larger strains compared to the filled system, with the critical strain found within $0.3-0.4$ of the strain range. In the case of CNTpolycarbonate composites, the critical strain decreases with increasing CNT content, presumably due to the presence of a filler network superstructure. However, for the nanocomposites with CNT concentration up to $5 \mathrm{wt} \%$, the strain range below 0.01 can be taken as a safe one to study the linear viscoelastic behavior. Interestingly, the nanocomposites show the lower storage modulus than that of the unfilled polycarbonate melt at highstrain region, the tendency clearly increasing with the CNT content. Such behaviour can be presumably explained by interplay of two effects: 1) the filler network structure becomes totally destroyed at such high strains and does not contribute into the modulus, 2) local strains in the matrix should be effectively higher than the applied strains due to the presence of hard particles, i.e. due to the effect of strain amplification which was also observed in the case of low density polyethylene nanocomposites filled with layered double hydroxide particles [8].

The linear viscoelastic response of CNT-polycarbonate composites has been studied using the constant strain amplitude of $\gamma_{0}=0.005$ and frequency sweep from 0.01 to $100 \mathrm{rad} / \mathrm{s}$. In the case of unfilled
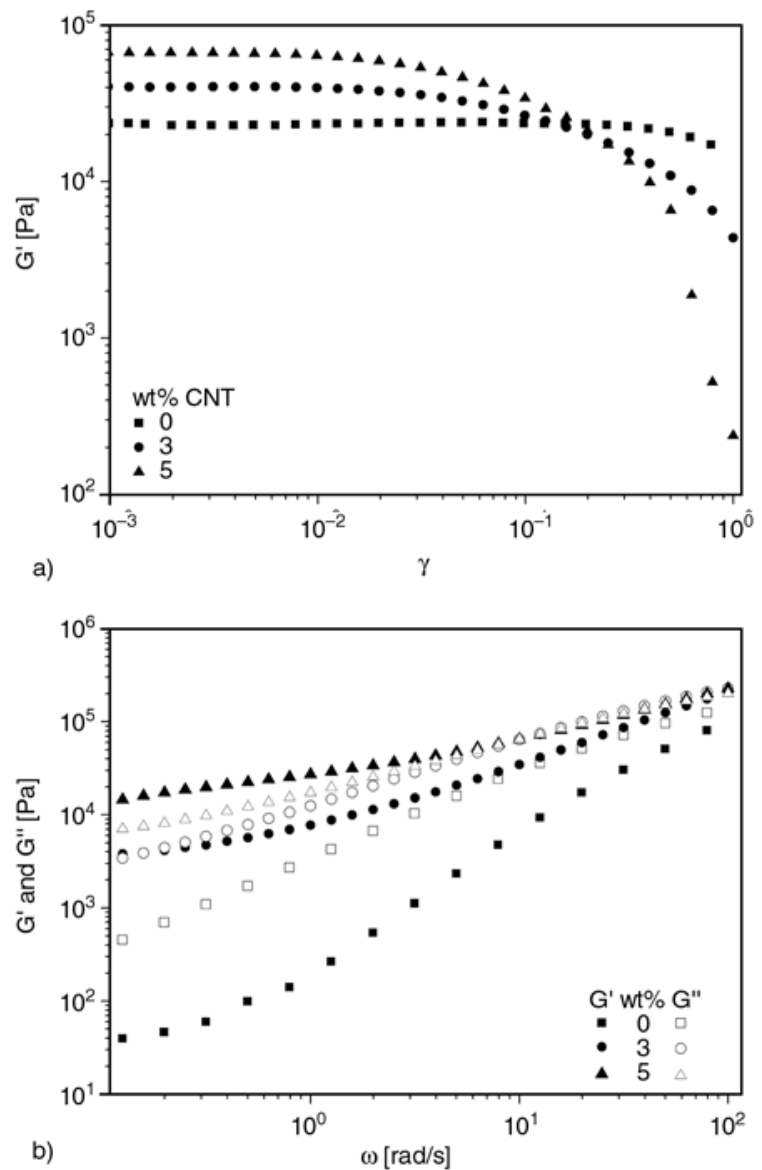

Figure 5. a) Strain amplitude sweeps for the storage modulus $G^{\prime}$ of CNT-polycarbonate composites. $\omega=$ $10 \mathrm{rad} / \mathrm{s} . T=230^{\circ} \mathrm{C}$. b) Frequency sweeps of the storage $G^{\prime}$ and loss $G^{\prime \prime}$ moduli of CNT-polycarbonate composites. $\gamma_{0}=0.005 . T=230^{\circ} \mathrm{C}$. 
polycarbonate, the storage modulus $G^{\prime}$ is found to be considerably lower than the loss modulus $G^{\prime \prime}$ within the experimental frequency range (Figure 5b). This means that the unfilled polycarbonate melt has dominant viscous character at $230^{\circ} \mathrm{C}$. Upon addition of $3 \mathrm{wt} \%$ CNTs, both moduli noticeably increase, nevertheless $G^{\prime}$ stays lower than $G^{\prime \prime}$ except the regions with the lowest and highest frequencies. Only when $5 \mathrm{wt} \%$ CNTs is added to the polymer matrix, the storage modulus becomes approximately three times larger than the loss modulus up to the frequencies of about $2 \mathrm{rad} / \mathrm{s}$. At higher frequencies $G^{\prime}$ and $G^{\prime \prime}$ have similar values.

Typically, the polymer melts filled with CNTs exhibit the transition from liquid-like to solid-like behavior at considerably lower values of the CNT loading, somewhere about 1 wt $\%$ [9, 10]. Presumably, the behavior observed is caused by very high shear rates during the injection molding resulting in a total destruction of the filler network structure, which is thought by most scientists to be responsible for the liquid-solid transition. This assumption is supported by the TEM images presented in Figure 1a which show the CNT clusters to be very small.

In the case of unfilled polycarbonate, the frequency sweeps from 0.01 to $100 \mathrm{rad} / \mathrm{s}$ were measured, additionally, at three different temperatures: 200, 250 and $300^{\circ} \mathrm{C}$. To obtain reliable data at higher temperatures, the strain amplitude was increased to 0.05 at $250^{\circ} \mathrm{C}$ and to 0.1 at $300^{\circ} \mathrm{C}$. The frequency sweeps at different temperatures have been used to construct the master curves at the reference temper-

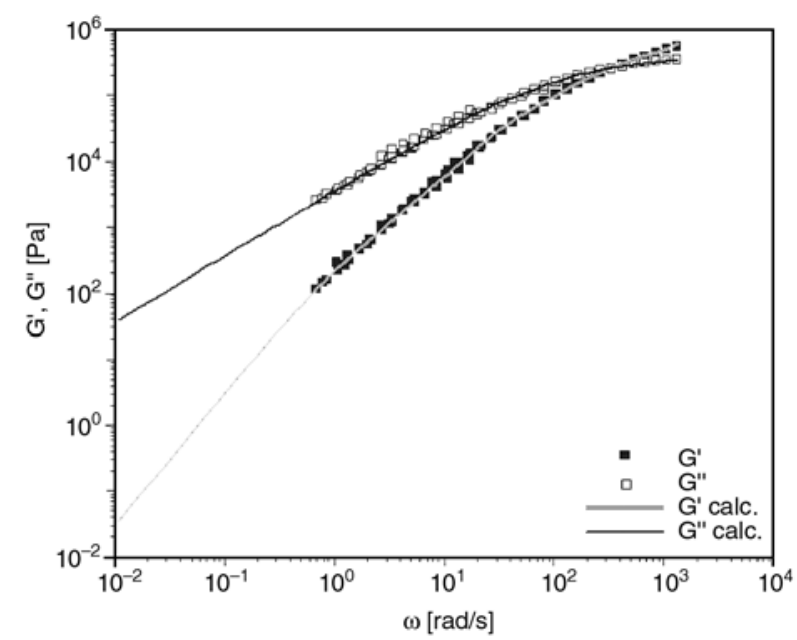

Figure 6. Master curves at $230^{\circ} \mathrm{C}$ obtained from the data measured at four different temperatures: 200, 230,250 and $300^{\circ} \mathrm{C}$. Also shown are the fits of $G^{\prime}$ and $G^{\prime \prime}$ obtained from conversion of the PC relaxation spectrum (see Table 1).
Table 1. Relaxation spectrum of $\mathrm{PC}$ at $230^{\circ} \mathrm{C}$

\begin{tabular}{|c|c|c|}
\hline $\mathbf{i}$ & $\mathbf{g}_{\mathbf{i}}[\mathbf{P a}]$ & $\boldsymbol{\lambda}[\mathbf{s}]$ \\
\hline 1 & $6.116 \cdot 10^{5}$ & $6.377 \cdot 10^{-4}$ \\
\hline 2 & $2.698 \cdot 10^{5}$ & $4.125 \cdot 10^{-3}$ \\
\hline 3 & $6.365 \cdot 10^{4}$ & $2.313 \cdot 10^{-2}$ \\
\hline 4 & $2.563 \cdot 10^{3}$ & $1.703 \cdot 10^{-1}$ \\
\hline 5 & $2.227 \cdot 10^{2}$ & $9.205 \cdot 10^{-1}$ \\
\hline
\end{tabular}

ature of $230^{\circ} \mathrm{C}$ (Figure 6). For further analysis, the master curves for $G^{\prime}(\omega)$ and $G^{\prime \prime}(\omega)$ for unfilled polycarbonate have been converted into the discrete relaxation time spectrum using the commercial software 'IRIS Rheo-Hub 2007' [15]. IRIS expresses the relaxation modulus $G(t)$ as a sum of $N$ Maxwell modes, as shown by Equation (1):

$G(t)=\sum_{i=1}^{N} g_{i} \exp \left(-\frac{t}{\lambda_{i}}\right)$

The weight coefficients, $g_{i}$, and the relaxation times, $\lambda_{i}$, in Equation (1) are determined by the Baumgärtel's method [16]. This method is based on two major premises. These are (1) the simultaneous fitting of $G^{\prime}$ and $G^{\prime \prime}$ in order to robust the numerical algorithm, and (2) the prevention of 'overfitting' by choosing the admissible number of modes per decade depending on the noise in the data.

Weight coefficients $g_{i}$ and relaxation times $\lambda_{i}$ of the discrete relaxation spectrum for the PC 2805 at $230^{\circ} \mathrm{C}$ are summarized in Table 1 . The longest relaxation time is about $1 \mathrm{~s}$ and the zero-shear viscosity is $\eta_{0}=3620 \mathrm{~Pa} \cdot \mathrm{s}$. It can be seen from Figure 6 that a back conversion from the time domain to the frequency domain provides a perfect fit of the storage and loss moduli.

\section{Model description}

If the polymer melt is filled with attractively interacting nanoparticles, its rheological behaviour can be relatively well described in the framework of superposition approach originally proposed by Leonov [17]. In this approach the total stress tensor, $\sigma$, is represented by a sum of two stresses, as shown by Equation (2):

$\boldsymbol{\sigma}=X \boldsymbol{\sigma}_{p}+\boldsymbol{\sigma}_{f}^{\mathrm{Net}}$

where $\sigma_{p}$ is the viscoelastic stress arising in the matrix due to orientation of polymer strands. This component is described in our study by the DoiEdwards model proposed for the linear polymer 
chains [18]. Hydrodynamic reinforcement of the polymer matrix is taken into account by multiplying $\sigma_{p}$ by the hydrodynamic amplification factor, $X$. In the case of highly elongated CNT particles, which we consider here in the first approximation as prolate spheroids, $X$ is given by Equation (3) [19]:

$X=1+2 \phi\left(1+\frac{A}{15}\right)$

Equation (3) is valid in the case of isotropic orientation of elongated particles, $\phi$ is the volume fraction of the filler particles and $A$ is the stress-shape coefficient (Equation (4)):

$$
A=\frac{r^{2}}{2 \ln r}, \quad r>>1
$$

which depends only on the particle aspect ratio $r=L / d$ ( $L$ is the particle length and $d$ is its diameter). The second component on the right side of Equation (2), $\boldsymbol{\sigma}_{f}^{\text {Net }}$, arises due to attractive interactions between the particles. It is described by a modified viscoelastic Maxwell model containing a structural parameter that reflects the state of the particle network at particular shear conditions [17]. Thus, we consider only hydrodynamic interactions between CNTs and the polymer matrix and neglect possible attractive interactions between them. Although the latter can be also taken in to account (for example using the formalism proposed by [20]), it will complicate the description considerably.

\subsection{Doi-Edwards model for large-strain viscoelasticity}

Doi and Edwards developed a constitutive equation for entangled polymer melts that combines the linear viscoelastic response for a reptating chain with a nonlinear response to large deformations [18]. The stress tensor of the Doi-Edwards (DE) theory with the assumption of independent alignment (IA) of the polymer strands is described by Equation (5):

$$
\boldsymbol{\sigma}_{p}(t)=\int_{-\infty}^{t} \frac{\partial G\left(t-t^{\prime}\right)}{\partial t^{\prime}} \mathbf{S}^{I A}\left(t, t^{\prime}\right) \mathrm{d} t, \quad \mathbf{S}^{I A}=5 \mathbf{S}_{2}
$$

where $G(s)$ is the stress relaxation modulus at time $s=t-t^{\prime}$ and $\mathbf{S}^{I A}$ is the nonlinear viscoelastic strain tensor. The factor of 5 is introduced into the definition so that $\mathbf{S}^{I A}$ equals the linear viscoelastic strain tensor $\gamma\left(t, t^{\prime}\right)$, when the strain is small. $\mathbf{S}_{2}$ has a meaning of the second-order orientation tensor of polymer strands (Equation (6)):

$$
\mathbf{S}_{2}=\int \frac{(\mathbf{E} \cdot \mathbf{u})(\mathbf{E} \cdot \mathbf{u})}{|\mathbf{E} \cdot \mathbf{u}|^{2}} \mathrm{du}
$$

where $\mathbf{u}$ is the unit vector parallel to the end-to-end vector of a strand and $\mathbf{E}\left(t, t^{\prime}\right)$ is the displacementgradient tensor [21]. It is important to note that the deformed vector $\mathbf{E} \cdot \mathbf{u}$ in Equation (6) is normalized on its magnitude. This means that in the original DE model the polymer strand is assumed to be oriented by the flow but not stretched.

In this study Equations (5) and (6) were solved using the commercial software IRIS Rheo-Hub 2007 [15]. First, we followed the approach proposed for polydisperse linear polymer melts [22]. In this approach, an actually measured relaxation spectrum for a particular polymer is used instead of the original DE expression for a reptational relaxation spectrum. However, if one takes the relaxation spectrum extracted from the master curves for PC 2805 , i.e. $\left\{g_{i}, \lambda_{i}\right\}$ given in Table 1 , it is impossible to predict the stress growth curves measured in a relatively narrow interval of shear rates, $0.05 \mathrm{~s}^{-1} \leq \dot{\gamma}_{0} \leq 1 \mathrm{~s}^{-1}$ (see Figure 7a). First, the theoretically predicted curves lay considerably lower than the experimentally measured ones. So, the value of stationary viscosity at $0.05 \mathrm{~s}^{-1}$, at the lowest shear rate used, is found to be about $8000 \mathrm{~Pa} \cdot \mathrm{s}$. This means that the value of zero-shear viscosity extracted from the stress growth experiment is approximately two times higher than that extracted from the linear data. Why PC 2805, the polycarbonate batch used in this study, exhibits such a discrepancy is presently not clear. Second, none of the predicted curves exhibits a shear overshoot observed clearly at least for two middle shear rates. Thus, it seems that the approach based on the original DE equation (Equation (5)) does not work well in the case of PC 2805, although the polydispersity effects have been taken into account via an actually measured relaxation spectrum. Recently, it has been shown that a very good description of the experimental data of polydisperse polyethylene melts can be obtained in the frame of molecular stress function (MSF) theory which accounts for the process of convective constraint release [23]. 
In the following, we will restrict ourselves to the simplified description of matrix behaviour based on the original DE model with the single relaxation mode $g_{1}=8 \cdot 10^{4} \mathrm{~Pa}$ and $\lambda_{1}=0.1 \mathrm{~s}^{-1}$. This relaxation mode with the zero-shear viscosity of $\eta_{0}=8000 \mathrm{~Pa} \cdot \mathrm{s}$ describes reasonably well the overall course of stress growth curves, although it is not able to predict the shear overshoots observed at middle shear rates (see Figure $7 b$ ). Nevertheless, we will use this single relaxation mode for the description of transient viscosity, $\eta_{p}$, of the polycarbonate matrix in the stress growth experiment with filled systems. The transient viscosity is defined by Equation (7) as the ratio of shear component, $\sigma_{p}$, of the stress tensor $\boldsymbol{\sigma}_{p}$ (Equation (5)) to the constant shear rate, i.e. (Equation (7)):
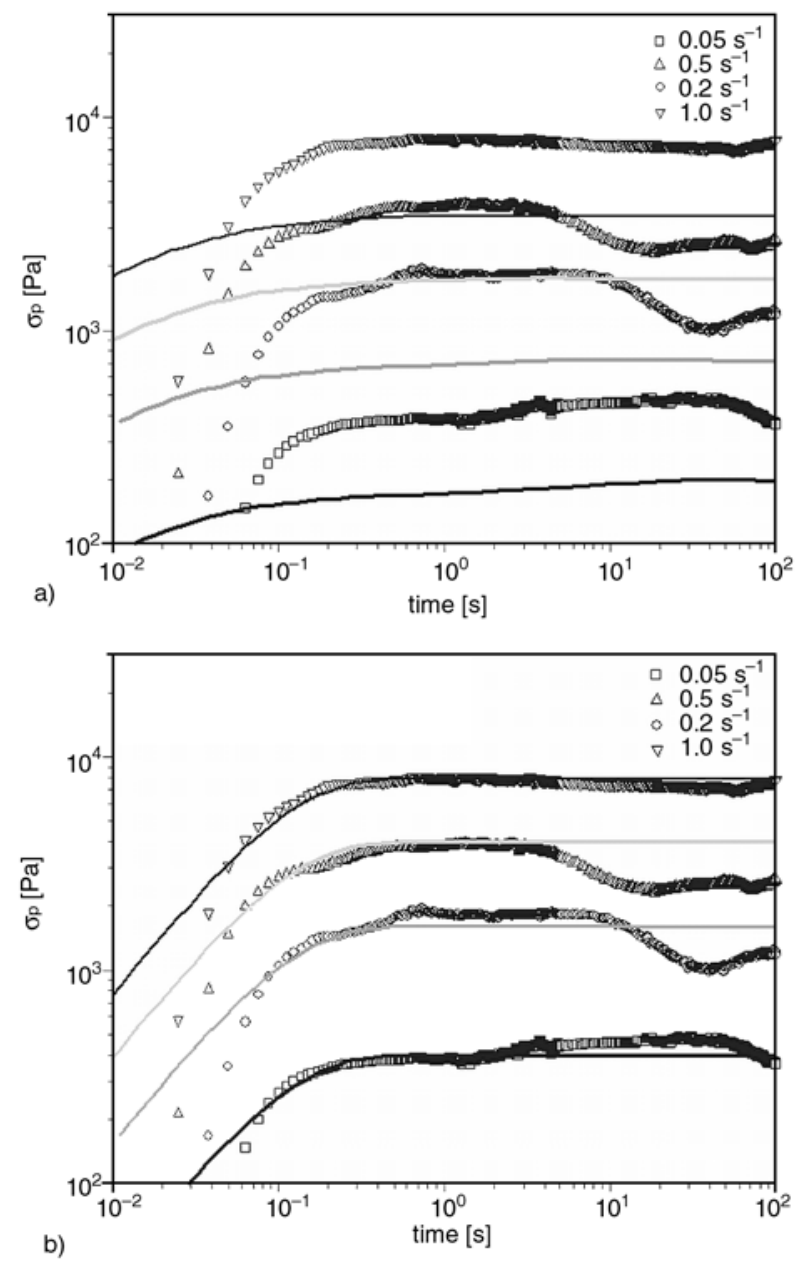

Figure 7. a) Stress growth curves for unfilled PC at four different shear rates: open symbols - experiment, lines - fit using Equation (5) and the relaxation spectrum given by Table 1 . b) Stress growth curves for unfilled PC at four different shear rates: open symbols - experiment, lines fit using Equation (5) and the single relaxation mode (see text). $\eta_{p}(t)=\frac{\sigma_{p}(t)}{\dot{\gamma}_{0}}$

\subsection{Modified viscoelastic Maxwell model}

Let us consider the CNT network as a viscoelastic structure. We assume that the stress experienced by the filler structure can be described by a modified viscoelastic Maxwell model given by Equation (8):

$\left(\sigma_{f}^{N e t}\right)_{(1)}+\frac{G_{f}}{\eta\left(\xi_{b}\right)} \sigma_{f}^{\text {Net }}=G_{f} \dot{\gamma}$

where $\dot{\gamma}$ is the rate-of-strain tensor and $\left(\boldsymbol{\sigma}_{f}^{\text {Net }}\right)_{(1)}$ denotes the upper-convected time derivative of a tensor $\boldsymbol{\sigma}_{f}^{\text {Net }}$ [21]. Here $G_{f}$ is the effective elastic modulus of the filler network. Usually, it is considerably lower than the Young modulus of one filler particle. For example, the Young modulus of about 0.2-1 TPa has been reported for single carbon nanotubes [24], while in the present study $G_{f}$ is found to be of about $50 \mathrm{kPa}$. The term $\eta$ is the effective viscosity of the filler network which depends on the structural parameter $\xi_{b}$. Thus, Equation (8) implies a process-dependent relaxation time which is an intrinsic feature of the thixotropic systems [25, 26]. The modified Maxwell model provides a phenomenological description of the structural rearrangements in the filler network structure. It is however physically motivated. To ascribe a simple physical meaning to $\xi_{b}$, let us assume that the filler structure is stabilized by a number of physical bonds that can be broken by the shearing in a nonlinear regime and can be again re-built during the quiescent time. These physical bonds represent attractive interactions between the multi-walled carbon tubes being part of the percolating filler network which bears a stress. This simple physical interpretation for example implies that the irreversible attractive interactions in primary agglomerates should not contribute into the structural parameter. Further, it is assumed that the total number of bonds - broken and unbroken - is not changing. Hence, a usual kinetic equation (Equation (9)) can be written for the change of the fraction of broken bonds $\xi_{b}$ :

$\frac{\mathrm{d}}{\mathrm{d} t} \xi_{b}=a\left|\dot{\gamma}_{0}\right|\left(1-\xi_{b}\right)^{n}-\lambda_{0}^{-1} \xi_{b}$

where the first term on the right side describes the bond rupture in a flow with the shear rate $\dot{\gamma}_{0}$, while the second term allows for the bond re-building 
driven by strong attractive interactions between the particles. Here $a$ is the rupture strength and $\lambda_{0}$ is the relaxation time in the quiescent state. The exponent $n$ describes the order of the kinetic process. We tried both the first-order kinetics with $n=1$ and the second-order kinetics with $n=2$ and obtained very similar results. Therefore, for the sake of simplicity, we consider further only the case with $n=1$.

On the start-up of shear flow, the fraction of broken bonds increases according to Equation (10):

$\xi_{b}(t)=a \dot{\gamma}_{0} \lambda_{\gamma}\left(1-e^{\frac{-t}{\lambda_{\gamma}}}\right)$ with $\lambda_{\gamma}=\frac{\lambda_{0}}{1+a \dot{\gamma}_{0} \lambda_{0}}$

where $\lambda_{y}$ is the relaxation time in the presence of flow. During the relaxation process, when the flow is switched off, the time dependence is given by Equation (11):

$\xi_{b}(t)=a \dot{\gamma}_{0} \lambda_{\gamma} e^{\frac{-t}{\lambda_{0}}}$

In the nonlinear regime, i.e. at high shear rates, $\lambda_{\gamma}$ may become significantly lower than $\lambda_{0}$ which results in two totally different time scales in the system [27].

The time-dependent effective viscosity can be now defined by Equation (12):

$$
\eta\left(\xi_{b}\right)=\frac{\eta_{f}}{m\left(\xi_{b}(t)\right)}
$$

where $\eta_{f}$ is the viscosity parameter and $m\left(\xi_{b}\right)$ is the mobility function. There are different ways to define the mobility function in literature [26, 28]. To choose an appropriate one, it is important to satisfy a number of criteria:

1) Highly filled polymer nanocomposites exhibit the transition to solid-like behavior in the limit of vanishing shear rates. A typical manifestation of such transition is the appearance of low-frequency plateau for the storage modulus $G^{\prime}$ on Figure $5 b$. Hence, $m\left(\xi_{b}\right) \rightarrow 0$ if $\xi_{b} \rightarrow 0$.

2) At high shear rates, a typical viscoelastic liquid behavior is observed. Hence, $\eta\left(\xi_{b}\right)$ should be a decreasing function of the structural parameter, i.e. $\mathrm{d} m\left(\xi_{b}\right) / \mathrm{d} \xi_{b}>0$.

The following form, given by Equation (13), proposed by [29]: $m\left(\xi_{b}\right)=\frac{\left(1-\xi_{b}\right)^{-d}-1}{d}, \quad d>0$

meets the above criteria. Equation (13) predicts a perfect elastic response of the undamaged filler structure, i.e. the loss modulus of filler structure $G_{f}^{\prime \prime}$ vanishes at $\omega \rightarrow 0$. This is hardly the case as the loss modulus of highly filled polymer nanocomposites considerably exceeds that of a polymer matrix at small and intermediate frequencies and even exhibits a kind of low-frequency plateau as can be seen from Figure $5 \mathrm{~b}$. To describe a non-vanishing $G_{f}^{\prime \prime}$ at small frequencies, one should add to the left side of Equation (13) the residual parameter $\alpha$ [17], see Equation (14):

$$
m\left(\xi_{b}\right)=\alpha+\frac{\left(1-\xi_{b}\right)^{-d}-1}{d}, \quad \alpha<1
$$

This expression for mobility function will be used in the modeling of stress growth curves as well as of time-dependent viscoelastic moduli in the flocculation experiment.

To calculate the latter let us consider the oscillatory shearing described by Equation (15):

$\gamma=\gamma_{0} \sin \omega t$

The changes in the structural parameter $\xi_{b}(t)$ can be calculated using Equations (10) and (11) in which instead of $\dot{\gamma}_{0}$ one should put the effective shear rate (Equation (16)):

$\dot{\gamma}_{e f f}=\frac{2}{\pi} \gamma_{0} \omega$

defined as an average over half period of the instantaneous shear rate $\dot{\gamma}=\gamma_{0} \omega \cos \omega t$. Thus, we neglect small oscillatory fluctuations of the structural constant around its stationary value, as they do not influence the values of $G_{f}^{\prime}$ and $G_{f}^{\prime \prime}$ that are the averages over at least one oscillation period $T=2 \pi / \omega$. Let us now rewrite Equation (8) for the shear component of $\boldsymbol{\sigma}_{f}^{\text {Net }}$ in the case of oscillatory shearing, obtaining Equation (17):

$$
\frac{\mathrm{d}}{\mathrm{d} t} \sigma_{f}^{N e t}+\frac{m\left(\xi_{b}\right)}{\tau_{f}} \sigma_{f}^{N e t}=G_{f} \gamma_{0} \omega \cos \omega t
$$

Here we made use of Equation (13) and introduced $\tau_{f}=\eta_{f} / G_{f}$. The time-dependent solution of Equation (17) can be found in the form of Equation (18): 
$\left.\sigma_{f}^{N e t}=\gamma_{0}\left\{G_{f}^{\prime}(t) \sin \omega t+G_{f}^{\prime \prime} t\right) \sin \omega t\right\}$

using the Matlab software. The stationary values of $G_{f}^{\prime}$ and $G_{f}^{\prime \prime}$, which are measured in the strain or frequency sweep experiments, can be defined analytically using the stationary value of the structural parameter, given by Equation (19):

$\xi_{b}\left(\gamma_{0}, \omega\right)=\frac{a \dot{\gamma}_{e f f}}{a \dot{\gamma}_{e f f}+\lambda_{0}^{-1}}$

This results in Equations (20):

$G_{f}^{\prime}=G_{f} \frac{\Omega^{2}}{\Omega_{0}^{2}+\Omega^{2}} \quad$ and $\quad G_{f}^{\prime \prime}=G_{f} \frac{\Omega_{0} \Omega}{\Omega_{0}^{2}+\Omega^{2}}$

where $\Omega=\omega \tau_{f}$ is the dimensionless frequency and is given by Equation (21):

$\Omega_{0}=\alpha+\frac{\left(1+a \lambda_{0} \dot{\gamma}_{\text {eff }}\right)^{d}-1}{d}$

One can see that in the limit of very small strain amplitudes, i.e. when $\dot{\gamma}_{\text {eff }} \rightarrow 0, \Omega_{0} \rightarrow \alpha$, and thus both the storage and the loss modulus have a nonzero value.

\section{Results and discussion}

\subsection{Stress growth experiment}

Figure 8 shows comparison between the shear growth curves measured for unfilled and filled with $5 \mathrm{wt} \% \mathrm{CNTs}$ polycarbonate melt at $230^{\circ} \mathrm{C}$. To avoid long memory effects typical for highly filled polymer systems [8], a new sample has been taken for every measurement and annealed during 15 minutes at $230^{\circ} \mathrm{C}$ before application of the shear flow. As expected, at all shear rates the transient stress for the filled system considerably exceeds that for the polymer matrix. This reinforcement effect is especially pronounced at the smallest shear rate used, i.e. $\dot{\gamma}_{0}=0.05 \mathrm{~s}^{-1}$. Further, we found out that the viscosity of filled PC increases monotonically with time for all shear rates except that of $0.5 \mathrm{~s}^{-1}$ (the weak maximum observed at this shear rate is most likely an experimental artefact), reaching a stationary value after the strain of about 1 (Figure 8). This is a potentially significant finding that deserves a careful consideration. Usually, in highly filled systems one observes a pronounced shear overshoot that can be ascribed to the orienta-

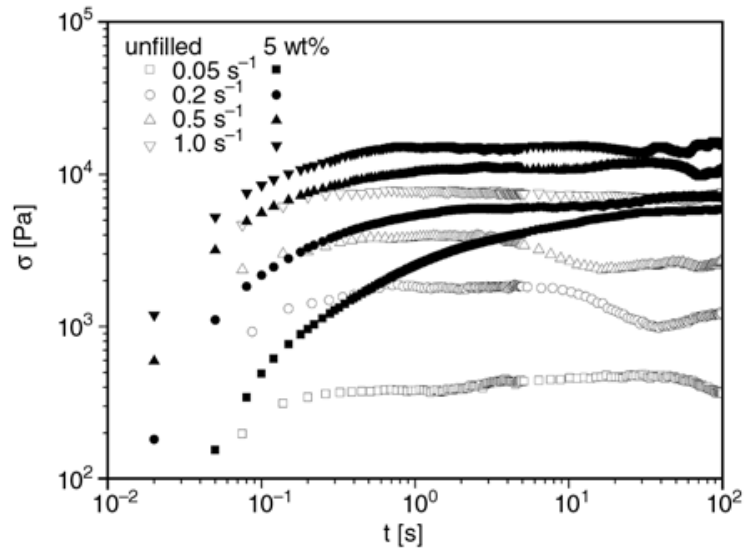

Figure 8. Stress growth curves at four different shear rates: open symbols - unfilled PC, filled symbols - PC filled with $5 \mathrm{wt} \%$ CNTs

tion of anisometric filler particles along the flow direction [7], or alternatively to the breakage of filler network structure under shear [8].

Let us first consider the orientation scenario. The morphological characterization with the help of $\mathrm{X}$-ray scattering and TEM studies provides the following information about the orientation state of CNTs after the injection molding. TEM images show no visible orientation of CNTs in the injection-molded plates (Figure 1a), even near the plate surfaces where the shear rates can be as high as $10^{3}-10^{4} \mathrm{~s}^{-1}$. According to the X-ray measurements the CNTs are only slightly oriented in the plane of the injection-molded plate, i.e. after application of extremely high shear rates. Therefore, we assume that there is no further orientation of CNTs in the ARES rheometer, i.e. under relatively small shear rates up to $1 \mathrm{~s}^{-1}$. Hence, we can use a constant value of the hydrodynamic amplification factor $X$ (the ratio of stationary viscosity of the filled system to that of the pure polymer matrix) given in the case of isotropic orientation of elongated particles by Equation (3).

The best fit of the upper curve $\left(\dot{\gamma}_{0}=1.0 \mathrm{~s}^{-1}\right)$ in Figure 9a is obtained if one takes the particle aspect ratio of about 35 . This corresponds to a value of the reinforcement factor $X \approx 1.9$ (Equation (3)). Thus, we have shown that the hydrodynamic reinforcement is relatively small for these polycarbonate composites filled with $5 \mathrm{wt} \%$ of CNTs. Although, one obtains a very good agreement between the predicted and measured data at $\dot{\gamma}_{0}=1.0 \mathrm{~s}^{-1}$, the discrepancy between the theory and the experiment increases with decrease of the shear rate. This can 

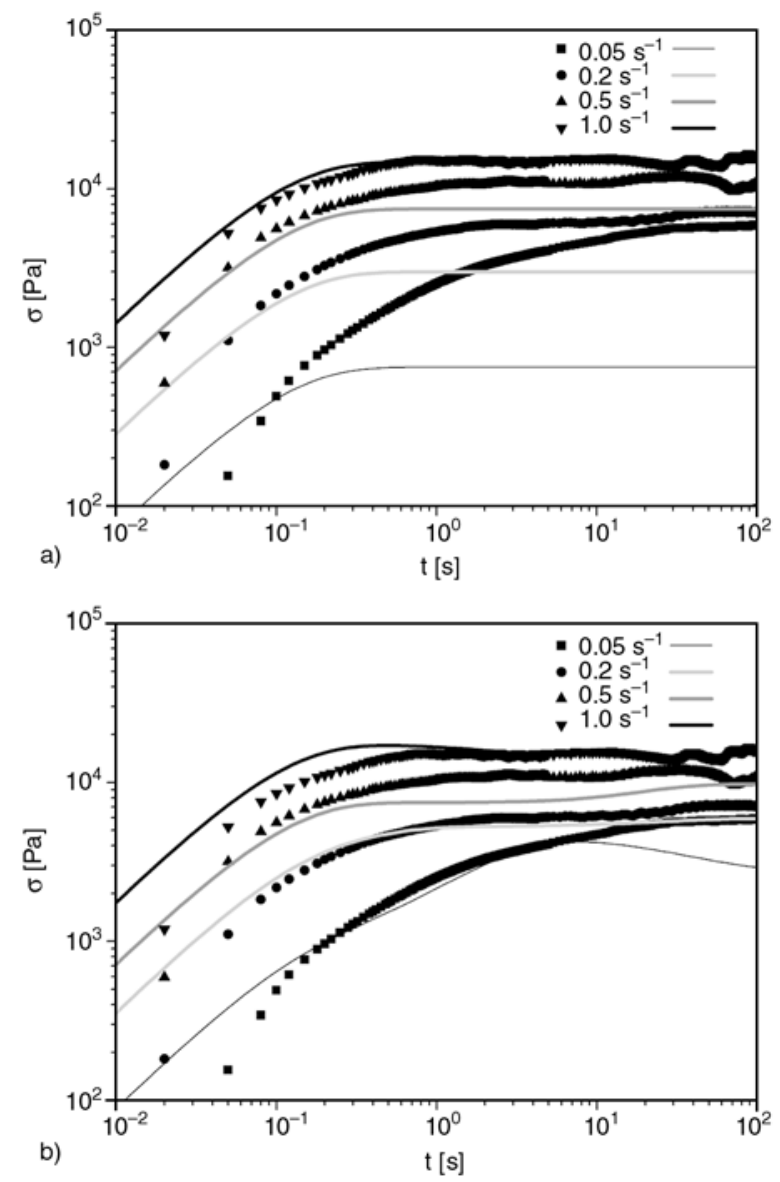

Figure 9. Stress growth curves for the PC filled with $5 \mathrm{wt} \% \mathrm{CNTs}$ at four different shear rates: filled symbols - experiment, lines - fit using Equation (8). $r=35$. CNT agglomeration is neglected (a), taken into account (b).

be explained by the presence of CNT agglomerates that either survived the process of injection molding or were formed in the injection-molded samples during the thermal treatment in the ARES cell $\left(15\right.$ minutes at $\left.230^{\circ} \mathrm{C}\right)$. At the smallest shear rate used, $\dot{\gamma}_{0}=0.05 \mathrm{~s}^{-1}$, the agglomerates cannot be effectively destroyed and thus they contribute into the total stress via the component $\boldsymbol{\sigma}_{f}^{\text {Net }}$ described by Equation (8). With the increase of shear rate, $\boldsymbol{\sigma}_{f}^{\text {Net }}$ decreases and becomes negligible at $\dot{\gamma}_{0}=1.0 \mathrm{~s}^{-1}$.

The best fit of shear growth curves in the presence of CNT agglomeration is shown in Figure 9b. Here we used a set of parameters extracted from the fitting of the 'flocculation' experiment that will be discussed in the following section. The relevant parameter for our discussion is the initial value of structural parameter $\xi_{b}$ which is taken here to be equal to 0.4 . This means that $40 \%$ of physical bonds between CNTs are broken in the composite samples which were annealed during 15 minutes at $230^{\circ} \mathrm{C}$ before shearing in the ARES cell. To achieve a fully relaxed initial state with $\xi_{b}=0$, one needs to anneal the sample at least two hours at $230^{\circ} \mathrm{C}$ prior application of the shear flow (see the next section). Figure $9 \mathrm{~b}$ indicates a satisfactory agreement between the predicted and measured data, with the only exception that the predicted transient shear stress slightly decreases at longer times for the curve with $\dot{\gamma}_{0}=0.05 \mathrm{~s}^{-1}$. Obviously, this slight decrease is a manifestation of breakage of CNT agglomerates.

\subsection{Flocculation experiment}

The dynamic oscillatory shearing at constant frequency shows that at low strain amplitudes both storage and loss moduli of the CNT/polycarbonate composites remain constant and independent of the applied strain (Figure 10), whereas above a critical strain $\gamma_{c r} \sim 0.01$ both moduli decrease with increasing strain. At two applied frequencies, $G^{\prime}$ decreases faster than $G^{\prime \prime}$. So, at $\omega=10 \mathrm{rad} / \mathrm{s}$, below $\gamma_{c r}$ the loss modulus is found to be only slightly lower than the storage modulus, whereas above $\gamma_{c r}$ it becomes noticeably higher than the storage modulus. At $\omega=1 \mathrm{rad} / \mathrm{s}$, this transition to the more viscous behavior takes place at a higher value of strain amplitude $\gamma_{0} \sim 0.1$ as the initial value of $G^{\prime}$ at this frequency is 1.5 times higher than that of $G^{\prime \prime}$. In the following $\omega=1 \mathrm{rad} / \mathrm{s}$ has been chosen to study the structural changes in the filled polycarbonate melt by subjecting it to different strain amplitudes, below and above the critical strain. An investigation which will be addressed shortly as the "flocculation' experiment.

At the first stage of the flocculation experiment an injection-molded sample is subjected to the oscillatory shearing with a strain amplitude $\gamma_{0}=0.005$. It is chosen to be so low that the sample at this stage can be considered to be in the quasi-quiescent state. The slight oscillatory perturbation is only necessary to detect the changes in $G^{\prime}$ and $G^{\prime \prime}$ with time. Thus, at the first stage one is able to follow in-situ the reagglomeration of the CNT clusters destroyed previously in the process of injection molding. When the stationary state is nearly attained, the sample is subjected to shearing with high strain amplitude at which the CNT clusters are effectively destroyed. Here, we chose a two-step shear-induced breakage of the filler structure, first at $\gamma_{1}=0.05$ and then at 

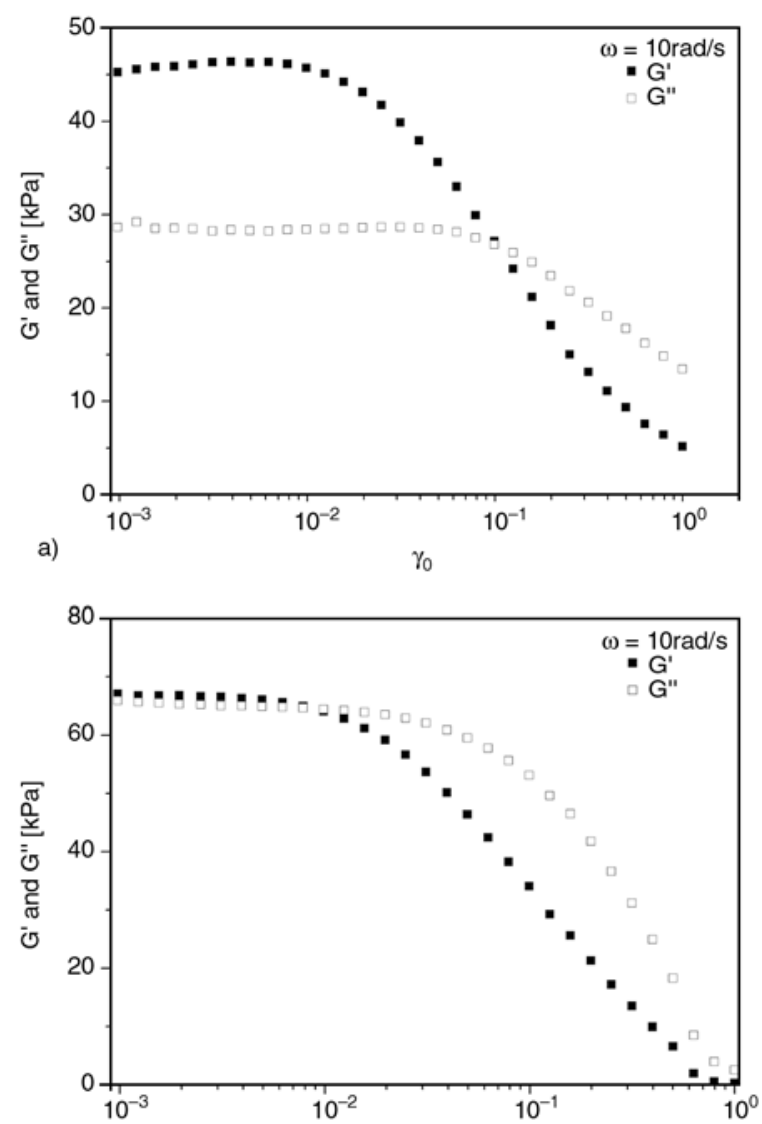

b)

$\gamma_{0}$

Figure 10. Strain amplitude sweeps of the storage $G^{\prime}$ and loss $G^{\prime \prime}$ moduli of PC filled with $5 \mathrm{wt} \%$ CNTs at two different frequencies: $\omega=1 \mathrm{rad} / \mathrm{s}$ (a) and $\omega=10 \mathrm{rad} / \mathrm{s}$ (b). $T=230^{\circ} \mathrm{C}$.

$\gamma_{2}=0.25$. For control, after attaining the stationary state at $\gamma_{2}=0.25$, the sample is again allowed to rest at the strain amplitude $\gamma_{0}=0.005$. It is meaningfully to characterize each shearing step by its effective shear rate $\dot{\gamma}_{\text {eff. }}$ In the flocculation experiment with $\omega=1 \mathrm{rad} / \mathrm{s}, \gamma_{0}=0.005$ corresponds to $\dot{\gamma}_{\text {eff }} \approx$ $0.003 \mathrm{~s}^{-1}, \gamma_{1}=0.05$ to $\dot{\gamma}_{\text {eff }} \approx 0.03 \mathrm{~s}^{-1}$ and $\gamma_{2}=0.25$ to $\dot{\gamma}_{\text {eff }} \approx 0.16 \mathrm{~s}^{-1}$.

Let us first consider the sample behavior in the quasi-quiescent state $\left(\gamma_{0}=0.005\right)$. Typical curves are presented on Figure 11. It can be seen that both moduli considerably increase with time: $G^{\prime}$ approximately three times and $G^{\prime \prime}$ approximately $25 \%$ compared to the initial values (Figure 11a). Further, one observes at least two recovery processes. The first process is relatively fast with the relaxation time of about $50 \mathrm{~s}$ and can be very likely associated with the agglomeration of CNTs into a sphere-like viscoelastic clusters [30]. Simple Brownian motion cannot be responsible for this agglomeration process as diffusion of non-interacting filler parti- cles is estimated to be too slow to be taken into account in the explanation of this effect. Rather, the strong attractive interactions between the dispersed carbon nanotubes and thermodynamic incompatibility between the particle and the polymer phases facilitate such fast agglomeration of the CNTs within a highly viscous medium [8]. The second recovery process is extremely slow without a sign of saturation even after two hours. It can be presumably associated with the gradual rearrangement of the CNT agglomerates in a network-like structure. Slow reorientation of CNTs back into the isotropic state cannot be responsible for this second recovery process in which the storage modulus increases on about $10 \mathrm{kPa}$. Such dramatic increase is far above the changes that can be caused in the storage modulus by the change in hydrodynamic reinforcement upon the reorientation of CNTs if one considers reasonable values of the aspect ratio, $r \leq 100$. After application of the oscillatory shearing with $\gamma_{1}=0.05$, the storage modulus drops immediately staying however higher than its initial value measured in the beginning of flocculation experiment (Figure 11a). The loss modulus stays unchanged which is in agreement with the data of strain amplitude sweep (Figure 10a). The fast drop in $G^{\prime}$ is caused solely by the rapid destruction of the CNT clusters as we can certainly exclude the orientation of tubes at this step with $\dot{\gamma}_{\text {eff }} \approx 0.03 \mathrm{~s}^{-1}$. As it was shown previously, there is no reorientation of CNTs at shear rates below $1.0 \mathrm{~s}^{-1}$.

The next shearing step with $\gamma_{2}=0.25$ is performed after the crossing point in Figure 10a and, therefore, the drop in both moduli leads now to a higher value of $G^{\prime \prime}$ compared to $G^{\prime}$ (Figure 11a). Interestingly, both moduli drop below their initial values measured in the beginning of flocculation experiment. The latter can be somewhat higher than the real initial values in the as-received probes, as the ARES software needs $8 \mathrm{~s}$ at $\omega=1 \mathrm{rad} / \mathrm{s}$ to measure the first point. Nevertheless, there is no doubt that the oscillatory shearing with $\gamma_{2}=0.25\left(\dot{\gamma}_{\text {eff }} \approx\right.$ $0.16 \mathrm{~s}^{-1}$ ) causes a similar qualitative change in the moduli as the injection molding. This is a very interesting finding as we can again prove that the orientation effects play a negligible role at $\dot{\gamma}_{\text {eff }} \approx$ $0.16 \mathrm{~s}^{-1}$ and thus the changes in moduli should be mainly assigned to the process of cluster destruction. Figure 11a shows that this destruction is very rapid and takes place at very small shearing rates. 



Figure 11. Flocculation experiment at different strain amplitudes: $\gamma_{0}=0.005, \gamma_{1}=0.05, \gamma_{2}=0.25$ and $\gamma_{3}=1.0, \omega=1 \mathrm{rad} / \mathrm{s}, T=230^{\circ} \mathrm{C}$. (a) PC filled with $5 \mathrm{wt} \%$ CNTs, (b) pure polycarbonate.

Obviously, this indicates that the CNT clusters are very fragile and thus can be easily damaged at shear rates $\dot{\gamma}_{0} \geq 1 \mathrm{~s}^{-1}$. Taking into account extremely high shear rates $\left(10^{3}-10^{4} \mathrm{~s}^{-1}\right)$ at the injection molding process, we assume a total breakage of clusters in the as-received samples.

The control step made at the quasi-quiescent conditions $\left(\gamma_{0}=0.005\right)$ shows a very similar behavior to the behavior at the first stage of the flocculation experiment. First of all, this means that all of the processes taking place in our system are fully reversible. Secondly, we can use this control step, together with two breakage steps preceding it, to test our superposition model as in all three cases we have a well-defined starting point. Before doing that, we have performed an additional control experiment with the unfilled polycarbonate (Figure 11b). At the quasi-quiescent conditions, a slight increase of both moduli has been found for the injection-molded sample. Application of the oscillatory shearing with $\gamma_{3}=1\left(\dot{\gamma}_{\text {eff }} \approx 0.67 \mathrm{~s}^{-1}\right)$ leads to a noticeable decrease of both moduli approximately to their initial values in the beginning of control experiment. During the second rest period the sample exhibits the same behavior as during the first rest period. In the following modeling of flocculation experiment, the gradual changes in the storage and loss moduli of the polycarbonate matrix will be neglected as these changes do not exceed $0.1 \mathrm{kPa}$ for $G_{p}^{\prime}$ and $1 \mathrm{kPa}$ for $G_{p}^{\prime \prime}$ as can be seen in Figure $11 \mathrm{~b}$. This is considerably lower than the corresponding changes, about $30 \mathrm{kPa}$ for $G^{\prime}$ and $10 \mathrm{kPa}$ for $G^{\prime \prime}$ (Figure 11a), caused by the structural rearrangement of the CNT-network. Therefore, the storage and loss moduli will be described in the first approximation by their average values: $G_{p}^{\prime}=200 \mathrm{~Pa}$ and $G_{p}^{\prime \prime}=3500 \mathrm{~Pa}$. The gradual changes in the total moduli are defined in the frame of superposition approach by the Equations (22) and (23):

$$
\begin{aligned}
& G^{\prime}(t)=X G_{p}^{\prime}+G_{f}^{\prime}(t) \\
& G^{\prime \prime}(t)=X G_{p}^{\prime \prime}+G_{f}^{\prime \prime}(t)
\end{aligned}
$$

Here, the hydrodynamic reinforcement of the polymer matrix is taken into account via the factor $X \approx 2$ that was extracted earlier from the fitting of stress growth curves.

In the modeling of flocculation experiment, the extremely slow relaxation process seen in Figure 11a will be neglected. Thus, we will concentrate on the phenomenological description of the agglomeration - de-agglomeration process responsible for the fast changes in the viscoelastic moduli. This process will be described by the modified viscoelastic Maxwell model which is characterized by a set of six parameters. Note, that it is necessary to introduce at least two more parameters, i.e. an additional kinetic equation (Equation (9)), if one would like to describe also the second (slow) relaxation process.

Such a large number of parameters is quite common in the modeling of thixotropic behavior [7, 25, 26]. The set of six parameters is the smallest one that is required to describe, even in a simplified manner, the physical phenomena that govern the structural kinetics and the rheology of CNT-polymer composites under study. Two of the model parameters, the relaxation time in the quiescent state $\lambda_{0}$ and the rupture strength $a$ (see Equation (9)), determine the evolution of the structural parameter $\xi_{b}$ and thus the rates of structural reorganization under shear and in its absence. The relaxation time $\lambda_{0}$ can be directly 
estimated from exponential fitting of the recovery process (not shown here). One can also estimate the relaxation time in the presence of flow $\lambda_{\gamma}$ from the two breakage steps and thus, using the formula (16), the value of parameter $a \sim 0.1$. Another two parameters, the residual parameter $\alpha$ and the exponent $d>1$, are necessary to calculate the timedependent effective viscosity via the mobility function (see Equation (14)). As was discussed above, the values of $\alpha$ and $d$ strongly influence the form of strain and frequency sweeps. Further, we will keep the value of parameter $d=2$ which means that shearing at high shear rates is able to destroy the filler structure completely. The last two parameters, the effective modulus of the filler structure $G_{f}$ and the viscosity parameter $\eta_{f}$, or their ratio $\tau_{f}=\eta_{f} / G_{f}$ that is more convenient in use, define the magnitudes of $G_{f}^{\prime}$ and $G_{f}^{\prime \prime}$. The value of $G_{f}$ can be identified with the storage modulus of filler structure in the absence of shear and thus it should be about the stationary value of modulus, ca. $40 \mathrm{kPa}$, at $\gamma_{0}=0.005$. Thus, we have actually only two parameters, $\tau_{f}$ and $\alpha$, that should be defined by fitting the experimental data.

Figure 12 presents the best fit of two breakage steps and the second quasi-rest period in the flocculation experiment using the set of parameters presented in Table 2. Relaxation times in the presence of oscillatory shear flow are $\lambda_{\gamma}=43 \mathrm{~s}$ for $\gamma_{1}=0.05$ and $\lambda_{\gamma}=28 \mathrm{~s}$ for $\gamma_{2}=0.25$. It can be seen that the evolution of storage modulus is reproduced reasonably well, but still a big discrepancy between the experi-

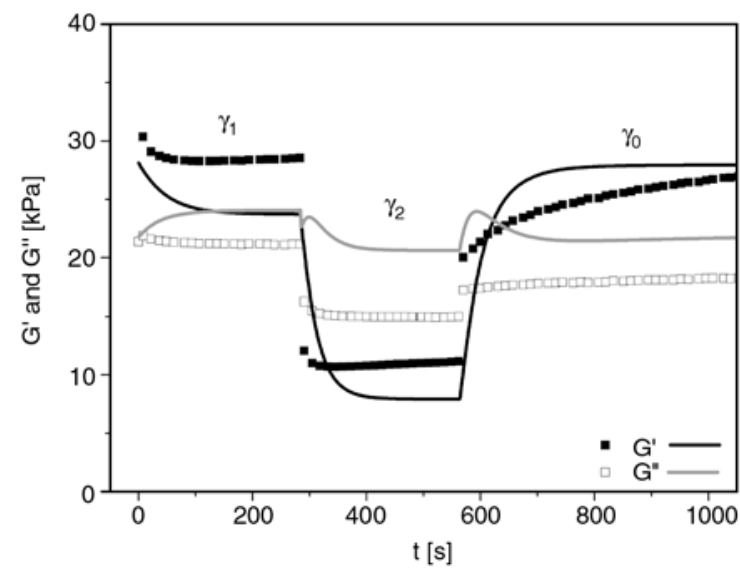

Figure 12. Time evolution of the storage $G^{\prime}$ and loss $G^{\prime \prime}$ moduli at different strain amplitudes: $\gamma_{0}=$ $0.005, \gamma_{1}=0.05$, and $\gamma_{2}=0.25, \omega=1 \mathrm{rad} / \mathrm{s}, T=$ $230^{\circ} \mathrm{C}$. Filled symbols - experiment, lines - fit using Equations (22) and (23) and the parameter set given in the Table 2 .
Table 2. Model parameters for PC filled with $5 \mathrm{wt} \%$ CNTs

\begin{tabular}{|l|c|}
\hline \multicolumn{1}{|c|}{ Parameter } & Value \\
\hline \multicolumn{2}{|c|}{ Matrix } \\
\hline Viscosity, $\eta_{0}$ Filler structure \\
\hline Reinforcement factor, $X$ & $8000 \mathrm{~Pa} \cdot \mathrm{s}$ \\
\hline \multicolumn{2}{|c|}{1.9} \\
\hline Relaxation time in the quiescent state, $\lambda_{0}$ & $50 \mathrm{~s}$ \\
\hline Rupture strength, $a$ & 0.1 \\
\hline Effective elastic modulus, $G_{f}$ & $35 \mathrm{kPa}$ \\
\hline Ratio $\tau_{f=} \eta_{f} / G_{f}$ & 0.8 \\
\hline Residual parameter, $\alpha$ & 0.4 \\
\hline Exponent, $d$ & 2 \\
\hline
\end{tabular}

mental data and theoretical prediction for the loss modulus exists. Similar problems were reported by [26], who could not provide a reasonable fit of the strain dependence of the loss modulus, although a number of elaborated viscoelastic models have been tried. Presently, we cannot say what process should be taken into account or how the model should be improved, to reach a better agreement with the experimental data for the loss modulus. Both moduli exhibit a noticeable jump in the moment of strain amplitude change, which is not predicted by the time-dependent solution of Equation (17). The same jump is exhibited by the PELDH nanocomposites upon the sudden change of strain amplitude, and thus it is not specific for the CNT-filled systems [8].

\section{Conclusions}

In this study we performed an extensive morphological and rheological characterization of the MWNT-polycarbonate composites produced by the injection molding technique. The main focus was to carry out such nonlinear viscoelastic experiments which allow to follow the structural rearrangements in the melt state.

In the first experiment, the polycarbonate composites with $5 \mathrm{wt} \%$ CNTs were sheared at a constant rate in the ARES rheometer to obtain a set of stress growth curves at different shear rates. The data obtained were then modelled using a superposition approach in which contributions from the polymer matrix, embedded nanotubes and their clusters have been accounted separately. The main effect found is the breakage of CNT clusters upon nonlinear shearing in the ARES rheometer. These clusters were presumably grown during 15 minutes tempering of as-received samples, as in the latter we 
expect a nearly total breakage of the CNT network structure in the process of injection molding.

In the second experiment, we followed agglomeration and deagglomeration processes by applying oscillatory deformation to the same composites. The strain amplitudes were chosen to be below and above the critical one, i.e. in linear and nonlinear viscoelastic regime. We found that both the storage and loss moduli of the as-received samples considerably increase in the linear regime. There are at least two recovery processes. One is relatively fast, with the relaxation time of about $50 \mathrm{~s}$, which corresponds presumably to the formation of carbon nanotubes agglomerates. The second process is extremely slow, with the relaxation time of several hours (a steady state is not achieved within the experimental time scale). This second recovery process is very likely caused by the gradual rearrangement of a whole network structure, although a definite conclusion can be only made after performing additional morphological studies, for example in-situ Rheo-SAXS measurements. When the strain amplitude is noticeably higher than the critical one, the network structure is effectively destroyed which results in a fast drop of both moduli, whereby the storage modulus becomes lower than the loss modulus. We have tried to reproduce the flocculation experiment in the frame of a modified Maxwell model capable of describing phenomenologically the transition from liquid-like to solid-like behavior in highly filled nanocomposites. The modified Maxwell model predicts reasonably well the time evolution of storage modulus, however there is a noticeable discrepancy between the theoretical description and the measured data for the loss modulus.

In overall, we can say that the main process influencing the viscoelastic properties of the MWNTpolycarbonate composites is the agglommerationdeagglommeration of nanotubes. Presumably, a main reason of suppression of the nanotube reorientation, both under shearing in the ARES rheometer and during the recovery process, is strong attractive interactions between the carbon nanotubes leading to their agglomeration into the viscoelastic clusters. We should admit that the modeling of aggregating filled systems, especially those composed from anisometric particles, is a difficult (maybe unsolved) task. However, we think that the superposition approach proposed here represents an essential step foreword as it can account not only for agglomeration but also for orientation effects which can become very pronounced during the melt processing of polymer nanocomposites. In the frame of superposition approach the orientation effects can be accounted for by using in Equation (2) instead of a constant reinforcement factor $X$ an additional contribution described by the Folgar-Tucker orientation model [7]. The modelling itself is very important in industrial applications of CNT based polymer composites and is planned to be implemented in commercial programs for description of the injection molding process.

\section{Acknowledgements}

This work was carried out with the support of the BMBF Project 'CarboNet' No. 03X0504E. Bayer Technology Services $\mathrm{GmbH}$ (Leverkusen, Germany) is greatly acknowledged for providing the samples for rheological measurements. Further, we would like to thank Dr. Klaus Schröter (Martin-Luther-University of Halle-Wittenberg, Germany) for very helpful discussions concerning the experimental rheological work. The authors are very grateful to $\mathrm{Mr}$. Torsten Hofmann (Leibniz-Institut für Polymerforschung Dresden e.V., Germany) for carrying out the X-ray scattering measurements. Dr. Petra Pötschke (Leibniz-Institut für Polymerforschung Dresden e.V., Germany) and Dr. Gilles Ausias and Mr. Ronan Simon (Université de Bretagne-Sud, Lorient, France) are greatly acknowledged for fruitful discussions.

\section{References}

[1] Nalwa H. S.: Polymeric nanostructures and their applications. American Scientific Publishers, Los Angeles (2007).

[2] Heinrich G., Klüppel M.: Recent advances in the theory of filler networking in elastomers. Advances in Polymer Science, 160, 1-44 (2002). DOI: $10.1007 / 3-540-45362-8 \quad 1$

[3] Fan Z., Advani S. G.: Rheology of multiwall carbon nanotubes suspensions. Journal of Rheology, 51, 585604 (2007).

DOI: $10.1122 / 1.2736424$

[4] Pegel S., Pötschke P., Petzold G., Alig I., Dudkin S. M., Lellinger D.: Dispersion, agglomeration, and network formation of multiwalled carbon nanotubes in polycarbonate melts. Polymer, 49, 974-984 (2008). DOI: 10.1016/j.polymer.2007.12.024

[5] Ray S. S., Okamoto M.: Polymer/layered silicate nanocomposites: A review from preparation to processing. Progress in Polymer Science, 28, 1539-1641 (2003).

DOI: $10.1016 /$ j.progpolymsci.2003.08.002 
[6] Moniruzzaman M., Winey K. I.: Polymer nanocomposites containing carbon nanotubes. Macromolecules, 39, 5194-5205 (2006).

DOI: $10.1021 / \mathrm{ma} 060733 \mathrm{p}$

[7] Lertwimolnun W., Vergnes B., Ausias G., Carreau P. J.: Stress overshoots of organoclay nanocomposites in transient shear flow. Journal of Non-Newtonian Fluid Mechanics, 141, 167-179 (2007).

DOI: $10.1016 / j . j n n f m .2006 .11 .003$

[8] Costa F. R., Saphiannikova M., Wagenknecht U., Heinrich G.: Layered double hydroxide based polymer nanocomposites. Advances in Polymer Science, 210, 101-168 (2008).

DOI: $10.1007 / 12 \quad 2007 \quad 123$

[9] Huang Y. Y., Ahir S. V., Terentjev E. M.: Dispersion rheology of carbon nanotubes in a polymer matrix. Physical Review B, 73, 125422/1-125422/9 (2006). DOI: 10.1103/PhysRevB.73.125422

[10] Pötschke P., Fornes T. D., Paul D. R.: Rheological behaviour of multiwalled carbon nanotube/polycarbonate composites. Polymer, 43, 3247-3255 (2002). DOI: $10.1016 / \mathrm{S} 0032-3861(02) 00151-9$

[11] Ren J., Silva A. S., Krishnamoorti R.: Linear viscoelasticity of disordered polystyrene-polyisoprene block copolymer based layered-silicate nanocomposites. Macromolecules, 33, 3739-3746 (2000).

DOI: $10.1021 / \mathrm{ma} 992091 \mathrm{u}$

[12] Solomon M. J., Almusallam A. S., Seefeldt K. F., Somwangthanaroj A., Varadan P.: Rheology of polypropylene/clay hybrid materials. Macromolecules, 34, 1864-1872 (2001).

DOI: $10.1021 / \mathrm{ma} 001122 \mathrm{e}$

[13] Liu A. J., Nagel S. R.: Nonlinear dynamics: Jamming is not just cool any more. Nature, 396, 21-22 (1998). DOI: $\underline{10.1038 / 23819}$

[14] Trappe V., Prasad V., Cipelletti L., Serge P. N., Weitz D. A.: Jamming phase diagram for attractive particles. Nature, 411, 772-775 (2001). DOI: $10.1038 / 35081021$

[15] Winter H. H., Mours M.: The cyber infrastructure initiative for rheology. Rheologica Acta, 45, 331-338 (2005). DOI: $10.1007 / \mathrm{s} 00397-005-0041-7$

[16] Baumgaertel M., Winter H. H.: Determination of discrete relaxation and retardatation time spectra from dynamic mechanical data. Rheologica Acta, 28, 511519 (1989). DOI: $10.1007 / \mathrm{BF} 01332922$

[17] Leonov A. I.: On the rheology of filled polymer melts. Journal of Rheology, 34, 1039-1068 (1990). DOI: $\underline{10.1122 / 1.550109}$

[18] Doi M., Edwards S. F.: The theory of polymer dynamics. Oxford University Press, Oxford (1988).
[19] Thomasset J., Carreau P. J., Sanschagrin B., Ausias G.: Rheological properties of long glass fiber filled polypropylene. Journal of Non-Newtonian Fluid Mechanics, 125, 25-34 (2005).

DOI: $10.1016 /$ j.jnnfm.2004.09.004

[20] Simhambhatla M., Leonov A. I.: On the rheological modeling of filled polymers with particle-matrix interactions. Rheologica Acta, 34, 329-338 (1995). DOI: $10.1007 / \mathrm{BF} 00367150$

[21] Bird R. B., Armstrong R. C., Hassager O.: Dynamics of polymeric fluids. Wiley, New York (1987).

[22] Bastian H.: Non-linear viscoealsticity of linear and long-chain branched polymer melts in shear and extensional flows. PhD thesis, Stuttgart University (2001).

[23] Wagner M. H., Rubio P., Bastian H.: The molecular stress function model for polydisperse polymer melts with dissipative convective constraint release. Journal of Rheology, 45, 1387-1412 (2001).

DOI: $10.1122 / 1.1413503$

[24] Lu J. P.: Elastic properties of carbon nanotubes and nanoropes. Physical Review Letters, 79, 1297-1300 (1997).

DOI: 10.1103/PhysRevLett.79.1297

[25] Dullaert K., Mewis J.: A structural kinetics model for thixotropy. Journal of Non-Newtonian Fluid Mechanics, 139, 21-30 (2006). DOI: 10.1016/j.jnnfm.2006.06.002

[26] Yziquel F., Carreau P. J., Moan M., Tanguy P. A.: Rheological modelling of concentrated colloidal suspension. Journal of Non-Newtonian Fluid Mechanics, 86, 133-155 (1999). DOI: $\underline{10.1016 / \mathrm{S} 0377-0257(98) 00206-7}$

[27] Saphiannikova M., Costa F. R., Wagenknecht U., Heinrich G.: Nonlinear behaviour of the polyethylene/layered double hydroxide nanocomposites under shear flow. Polymer Science Series A, 50, 573-582 (2008). DOI: 10.1134/S0965545X0805012X

[28] Sobhanie M., Isayev A. I.: Modeling and experimental investigation of shear flow of a filled polymer. Journal of Non-Newtonian Fluid Mechanics, 85, 189-212 (1999). DOI: 10.1016/S0377-0257(99)00002-6

[29] Coussot P., Leonov A. I., Piau J. M.: Rheology of concentrated dispersed system in a low molecular weight matrix. Journal of Non-Newtonian Fluid Mechanics, 46, 179-217 (1993). DOI: $10.1016 / 0377-0257(93) 85046-\mathrm{D}$

[30] Alig I., Skipa T., Lellinger D., Bierdel M., Meyer H.: Dynamic percolation of carbon nanotube agglomerates in a polymer matrix: Comparison of different model approaches. Physica Status Solidi (b), 245, 2264-2267 (2008).

DOI: $\underline{10.1002 / p s s b .200879622}$ 